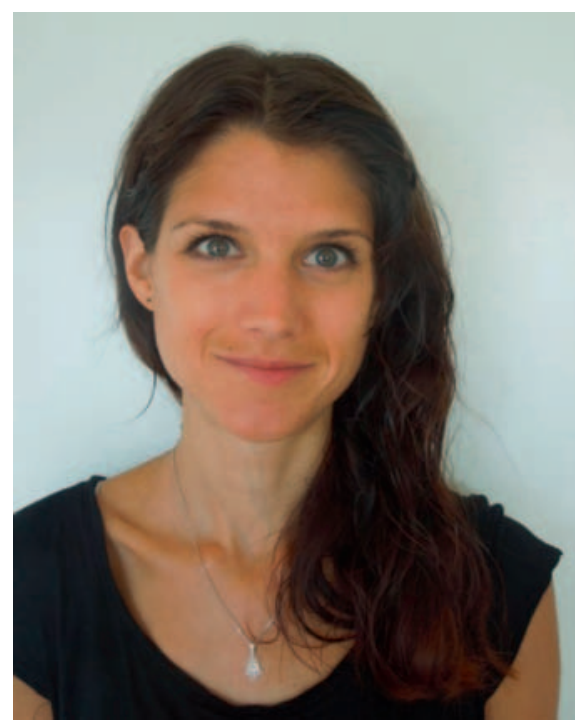

Sehr geehrte Frau Dr. Burgener, bitte stellen Sie uns doch den SDV kurz vor.

Der SDV wurde 1898 in Olten gegründet. Er bildet zusammen mit seinen Organen die nationale Branchenorganisation der Schweizer Drogerien. Heute sind rund 500 Drogerien in der Deutsch- und der Westschweiz Mitglieder des SDV. Das entspricht etwas mehr als $90 \%$ aller Schweizer Drogerien. Der Verband konzentriert seine Tätigkeiten auf die vier Geschäftsfelder «Politik und Branche», «Qualitätsmanagement», «Aus-, Fort-, Weiterbildung» und «Kommunikation und Medien« sowie auf den internen Bereich «Ressourcen und Services».

\title{
Drogisten und Komplementärmedizin
}

Frau Dr. sc. nat. Julia Burgener ist als Leiterin der Wissenschaftlichen Fachstelle des Schweizerischen Drogistenverbands (SDV) tätig. Der übergeordnete Zweck der Wissenschaftlichen Fachstelle ist der Schutz und die Förderung der drogistischen Interessen im Gesundheitswesen. Frau Dr. Burgener ist im Geschäftsfeld «Politik und Branche» auf der Umsetzungsebene aktiv - unter anderem bei der Konzeption und Durchführung von Bildungsmassnahmen im naturwissenschaftlichen Bereich. Ausserdem war sie von Ende 2010 bis Anfang 2014 als Post-Doc am Universitätsspital Zürich tätig, hat das Höhere Lehramt mit Zusatzqualifikation für Berufsschulen erreicht und ist diplomierte Yogalehrerin.

\section{Welche Rollen und Aufgaben übernimmt der SDV bisher insbesondere und welche Bereiche sollen eventuell in Zukunft dazukommen?}

Die Einflussnahme auf den politischen und gesetzlichen Gestaltungsprozess ist eine der Kernkompetenzen des Verbandes. Ziel ist es, für unsere Mitglieder optimale Rahmenbedingungen zu erwirken, damit diese ihren Beruf auch in Zukunft erfolgreich ausüben können. Im Bereich Aus-, Fortund Weiterbildung ist der SDV verantwortlich für die Bildungspläne der vierjährigen Grundbildung und des zweijährigen Vollzeitstudiums an der Höheren Fachschule für Drogistinnen und Drogisten. Ebenfalls bietet er seinen Mitgliedern ein umfassendes Weiterbildungsangebot an, damit die notwendige Beratungsqualität insbesondere auch im Bereich der Selbstmedikation und speziell auch der Komplementärmedizin jederzeit basierend auf aktuellem Know-how gewährleistet werden kann. Der SDV ist auch ein
Verlag. Dank unserem umfassenden Verbund elektronischer und gedruckter Medien erreichen die Drogerien ihre Kundinnen und Kunden regelmässig mit spannenden Informationen $\mathrm{zu}$ Gesundheit, Schönheit und Wohlbefinden. Seit 33 Jahren steht dabei der «Drogistenstern» im Zentrum, welcher mit 950000 Leserinnen und Lesern pro Ausgabe zu den grössten und renommiertesten Gesundheitszeitschriften der Schweiz gehört. Immer wichtiger wird zudem unsere Gesundheitsplattform vitagate.ch. Sie bietet monatlich aktuell rund 140000 Userinnen und Usern starke Inhalte zu verschiedenen Gesundheits- und Schönheitsthemen.

\section{Inwiefern sehen Sie eine Verbindung zwischen der Komplementärmedizin und den Drogisten? Wo gibt es Überschneidungen?}

Drogerien sind kompetente Abgabestellen im Bereich der Selbstmedikation und bieten dabei insbesondere

\section{KARGER}


auch eine fachkundige Beratung zu pflanzlichen und komplementärmedizinischen Arzneimitteln. Im Zentrum stehen dabei phytotherapeutische oder homöopathische Arzneimittel, spagyrische Essenzen, Bachblütentherapie, aber auch traditionelle asiatische Kräuter und Tees, Aromatherapie und Gemmotherapie. Damit die Drogerien bestmöglich auf die Bedürfnisse ihrer Kundinnen und Kunden eingehen können, sind sie auf eine möglichst grosse Heilmittelvielfalt in der Phytotherapie und restlichen Komplementärmedizin angewiesen.

\section{Welche Fort- und \\ Weiterbildungsmöglichkeiten \\ bestehen für Drogisten, die \\ sich vor allem für ganzheitliche \\ Ansätze bzw. pflanzliche \\ Heilmittel interessieren?}

Grundsätzlich hat die Ausbildung im Bereich der Phytotherapie und der Komplementärmedizin im Allgemeinen bereits während der achtjährigen Ausbildung zur Drogistin bzw. zum Drogisten HF eine grosse Bedeutung. Daneben ist das Fort- und Weiterbildungsangebot zu diesen Themen sowohl mit verschiedenen Kursen des SDV als auch mit Schulungen vonseiten der Hersteller phytotherapeutischer und komplementärmedizinischer Arzneimittel sehr breit.

\section{Wie wichtig finden Sie die Vernetzung innerhalb der Komplementärmedizin, d.h. zwischen Einzelpersonen, aber auch zwischen Verbänden und Gesellschaften?}

Welche Unterschiede bzw. Gemeinsamkeiten gibt es Ihrer

Ansicht nach zwischen der Schweiz und dem restlichen Europa?

Jede Vernetzung innerhalb der Komplementärmedizin trägt zu einem besseren Verständnis wie auch zu einer stärkeren Verankerung der Komplementärmedizin sowohl bei der breiten Bevölkerung als auch bei Fachpersonen bzw. Verbänden und Gesellschaften bei. Deshalb engagiert sich der Drogistenverband auch im Dachverband Komplementärmedizin (Dakomed). Die Abgabe von nicht verschreibungspflichtigen Arzneimitteln erfolgt in der Schweiz ausschliesslich durch Fach- und Medizinalpersonen. Daraus leitet sich das im europäischen Vergleich überdurchschnittlich hohe Vertrauen der Bevölkerung in die Selbstmedikation, auch gerade mit komplementärmedizinischen und pflanzlichen Arzneimitteln, ab. Unserem Gesundheitswesen bleiben dadurch viele Kosten erspart.

\section{Wie sind Sie selbst mit der Komplementärmedizin in Verbindung gekommen? Welche Pflanze bzw. welches Präparat/Extrakt fasziniert Sie insbesondere?}

Mein Weg zur Komplementärmedizin besteht genau genommen aus zwei verschiedenen Zubringern: Der erste Kontakt geschah mit der Anthroposophie im Kindergartenalter: So durfte ich regelmässig in die Eurythmie, wo ich lernte, Wasser oder Wellen darzustellen. Das hat mich un- glaublich fasziniert. Diese Ausbildungszeit hat für mich, im Nachhinein betrachtet, einen ganz wertvollen und wichtigen Beitrag für den Umgang mit der Natur und den Menschen geliefert. Der zweite Kontakt geschah dann nach dem Studium, während meiner Forschungsarbeit am Universitätsspital Zürich. Dort konnte ich direkt die Grenzen der Schulmedizin und die Möglichkeiten der Komplementärmedizin erleben. So weiss ich heute, dass die Komplementärmedizin Lücken der Schulmedizin zu schliessen vermag und sich diese zwei Richtungen daher optimal ergänzen.

Auch nach langem Nachdenken kann ich Ihnen kein konkretes Präparat oder Extrakt nennen, das mich fasziniert. Vielmehr bin ich immer wieder erstaunt über die vielfältige und heilende Wirkung von Gemüse. Die darin enthaltenen bioaktiven Stoffe können selbst in der Konzentration, wie sie in der täglichen Nahrung zu finden sind, vorbeugen und heilen.

Sehr geehrte Frau Dr. Burgener, herzlichen Dank für das Interview!

Interview: Alexander Eitner 